\title{
Evaluation of Antihypertensive Efficacy of Intravenous Hydralazine and Intravenous Labetalol in the Management of Severe Preeclampsia and Eclampsia
}

\author{
Shabana Yeasmin Siddika ${ }^{1}$, Sangita Patra ${ }^{2}$, Pallab Kumar Mistri ${ }^{3}$, Sanjib Das ${ }^{4}$ \\ 1Department of Obstetrics and Gynaecology, Medical College and Hospital, Kolkata, West Bengal, \\ India. ${ }^{2}$ Department of Obstetrics and Gynaecology, Medical College and Hospital, Kolkata, West \\ Bengal, India. ${ }^{3}$ Department of Obstetrics and Gynaecology, Medical College and Hospital, Kolkata, \\ West Bengal, India. ${ }^{4}$ Department of Obstetrics and Gynaecology, Medical College and Hospital, \\ Kolkata, West Bengal, India.
}

\section{ABSTRACT}

\section{BACKGROUND}

Hypertensive disorders of pregnancy are one of the most common obstetrical problems and affect $5-10 \%$ of all pregnancies. Severe preeclampsia is a multisystem disorder and causes many complications like intracranial haemorrhage, hypertensive encephalopathy, abruptio placentae, heart failure, pulmonary oedema and eclampsia. Intravenous hydralazine and labetalol are considered as first line antihypertensive agents for management of hypertensive crisis. The objective of this study was to compare efficacy of intravenous hydralazine and labetalol in lowering blood pressure in severe pregnancy induced hypertension, as well as to find out frequency of maternal adverse reactions and foetal impacts.

\section{METHODS}

The study was conducted at Calcutta National Medical College and Hospital over a period of one year. 100 women with severe preeclampsia were included in the study and randomly divided into two groups of 50 each. One group received intravenous hydralazine while the other group was treated with intravenous labetalol. The time and number of doses taken to achieve target blood pressure were noted. Number of patients having persistent severe hypertension after receiving maximum drug dosage were also taken into account.

\section{RESULTS}

The pre-treatment systolic blood pressure was $168.80 \mathrm{mmHg}$ in hydralazine group and $173.64 \mathrm{mmHg}$ in labetalol group whereas the diastolic blood pressure was $143.92 \mathrm{mmHg}$ and $142.6 \mathrm{mmHg}$ in hydralazine and labetalol group respectively. Mean time to achieve blood pressure control was 22.20 minutes in hydralazine group and 26.04 minutes in labetalol group. There were 3 cases of persistent severe hypertension in hydralazine group and 2 cases in labetalol group. No statistically significant difference was found between the two groups in terms of efficacy, maternal adverse effects and perinatal outcome. However, maternal tachycardia and headache was found to be significantly higher in patients treated with hydralazine.

\section{CONCLUSIONS}

Thus, both hydralazine and labetalol are effective and safe antihypertensive drugs which can be used to treat pregnancy induced hypertensive crisis. No significant difference is found between these two agents.

\section{KEY WORDS}

Severe Preeclampsia, Pregnancy Induced Hypertensive Crisis, Hydralazine, Labetalol
Corresponding Author:

Sangita Patra,

No. 82/7M, Ballygunge Place,

Kolkata-19, West Bengal, India.

E-mail: patra_sangita@yahoo.co.in

DOI: 10.14260/jemds/2020/209

Financial or Other Competing Interests: None.

How to Cite This Article:

Siddika SY, Patra S, Mistri PK, et al. Evaluation of antihypertensive efficacy of intravenous hydralazine and intravenous labetalol in the management of severe preeclampsia and eclampsia. J. Evolution Med. Dent. Sci. 2020;9(12):969-973, DOI: 10.14260/jemds/2020/209

Submission 16-01-2020,

Peer Review 27-02-2020,

Acceptance 04-03-2020,

Published 23-03-2020.

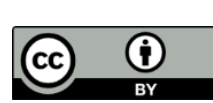




\section{BACKGROUND}

Pregnancy is a physiological phenomenon. However, some women develop problems during its evolution, putting both the mother's and the foetus's health at risk. Hypertensive disorders of pregnancy are one of the maternal diseases that cause the most detrimental effects to mother and foetus. Preeclampsia is a multisystem organ disorder unique to human pregnancy and despite decades of intensive research the exact pathology is still unknown. It still remains amongst the most important unresolved problems of obstetrics. It is one of the leading contributors of maternal morbidity and mortality in developed countries, more so in a developing country like India. Hypertensive disorders of pregnancy are one of the most common obstetrical problems and affects 5$10 \%$ of all pregnancies, being a major cause of maternal, foetal and neonatal morbidity and mortality.[1] Severe preeclampsia with blood pressure more than or equal to 160/110 mmHg affect multiple organ systems and associated with complications like hypertensive encephalopathy, intracranial haemorrhage, eclampsia, abruptio placenta, congestive heart failure and pulmonary oedema. ${ }^{[2]}$ Reduction in blood pressure to safe levels is necessary to prevent these complications. The obstetricians should aim not just for the diagnosis, but also to prevent the complications. Aggressive blood pressure control, while fundamental, needs to be balanced against the risks to both mother and foetus of overcorrection and under correction. Treatment of hypertensive crisis associated with hypertensive disorders of pregnancy remains under investigation. There are many studies and meta-analysis on the subject, but there is no definitive consensus or recommendation of great power regarding which is the best antihypertensive to achieve short term success in controlling of hypertensive crisis without affecting the wellbeing of the mother and the foetus. Intravenous labetalol and hydralazine are considered as first line antihypertensive agents for management of hypertensive crisis. ${ }^{[1,3]}$ Hydralazine is a directly acting smooth muscle relaxant acting as a vasodilator primarily in arteries and arterioles. Labetalol acts as a dual alpha 1 and beta adrenergic receptor blocker. It is highly selective for postsynaptic alpha 1 adrenergic and non-selective for beta adrenergic receptors. Labetalol acts as competitive antagonist at the receptor level and causes decrease in systemic arterial blood pressure and systemic vascular resistance without substantial decrease in resting heart rate, cardiac output and stroke volume.

The purpose of this study was to compare intravenous labetalol and hydralazine in regard to efficacy, time taken, and number of doses required to achieve target blood pressure and to assess any maternal and foetal complications.

\section{METHODS}

The study was conducted in the Department of Obstetrics and Gynaecology of Calcutta National Medical College and Hospital after obtaining ethical clearance from the Research Ethics Committee from July 2015 to June 2016. This prospective cohort study was done in 100 pregnant women who met the inclusion criteria and were allocated into 2 groups of 50 each after taking proper consent.

\section{Inclusion Criteria}

Patients with systolic BP more than or equal to $160 \mathrm{mmHg}$ and/or diastolic BP more than or equal to $110 \mathrm{mmHg}$ with pregnancy more than or equal to 28 weeks.

\section{Exclusion Criteria}

Patients with multifetal gestation, chronic hypertension, cardiac, liver and renal disease, anaemia and thalassemia, diabetes, asthma, allergic to labetalol or hydralazine, maternal heart rate $<60$ or $>120$ beats/minute.

\section{Methods of Data Collection}

The criteria for the diagnosis of severe preeclampsia were in accordance with the guidelines of the American College of Obstetricians and Gynaecologists. Standard mercury sphygmomanometer with appropriately sized cuff was used to measure blood pressure. The first and fifth Korotkoff sounds were recorded for systolic and diastolic blood pressure respectively. The blood pressure was measured with patient in recumbent position with the patient's arm at the level of the heart every time during measurements. Enrolled patients were subjected to clinical evaluation and laboratory investigations, i.e. complete blood count, peripheral smear, renal and liver function test, serum electrolytes, serum LDH (Lactate Dehydrogenase), coagulation profile, urine test. Patients allocated to Labetalol group were administered $20 \mathrm{mg}$ ( $4 \mathrm{ml}$ ) IV bolus slowly over 2 minutes. Maximum $220 \mathrm{mg}$ ( 4 doses) of labetalol was given in doses of 20,40,80,80 mg.[4] Blood pressure was recorded 10 minutes after each dose until the target BP, i.e. systolic blood pressure $<160 \mathrm{mmHg}$ and diastolic blood pressure $<110$ $\mathrm{mmHg}$ was achieved. Reconstitution of intravenous hydralazine was done by dissolving $20 \mathrm{mg}$ powder with $2 \mathrm{ml}$ $0.9 \%$ normal saline in the vial and further dilution done with $18 \mathrm{ml}$ normal saline which equates $1 \mathrm{mg} / \mathrm{ml}$ hydralazine. 5 mg IV bolus administered every 10 minutes up to a maximum of 4 doses. The time and number of doses required to achieve target blood pressure was noted. The blood pressure was recorded every 15 minutes till first 30 minutes after achieving target BP, then every 30 minutes for next 2 hours. All the patients were followed up for any adverse drug reaction. Continuous monitoring of maternal vital parameters and foetal heart rate was done. Mode of delivery and foetal outcome were recorded. Treatment was considered as failure and persistent severe hypertension was diagnosed if blood pressure did not decrease to satisfactory level even after increasing the dose to maximum.

Trial was terminated if there was successful lowering of blood pressure, patient developed hypotension, i.e. blood pressure less than or equal to $90 / 60 \mathrm{mmHg}$, excessive maternal tachycardia or bradycardia or foetal distress of any form. Management of severe preeclampsia included preventing seizures. All women initially received magnesium sulphate as a 4 gm intravenous dose over 10 minutes and 10 gm intramuscular ( $5 \mathrm{gm}$ in each buttock). Maintenance dose of $5 \mathrm{gm}$ in alternate buttocks was administered every 4 hours until 24 hours after delivery (Pritchard regimen). 


\section{Statistical Analysis}

Statistical analysis was done using chi square test and student's t test. Quantitative variables were indicated in mean and standard deviation. Probability value of less than 0.05 was accepted as indicating statistical significance.

\section{RESULTS}

\begin{tabular}{|cccc|}
\hline Parameters & Labetalol & Hydralazine & p Value \\
Age in years(Mean \pm SD) & $24.78 \pm 3.64$ & $24.86 \pm 3.99$ & 0.9168 \\
Gestational age in weeks (Mean \pm SD) & $36.88 \pm 2.75$ & $37.06 \pm 2.73$ & 0.7433 \\
SBP before treatment & $173.64 \pm 12.54$ & $168.80 \pm 14.60$ & 0.073 \\
(mmHg) [Mean \pm SD] & & & \\
DBP before treatment & $113.8 \pm 3.46$ & $113.04 \pm 3.57$ & 0.2825 \\
(mmHg) [Mean \pm SD] & $37(74 \%)$ & $35(70 \%)$ & \\
Booked & $13(26 \%)$ & $15(30 \%)$ & 0.6563 \\
Unbooked & $26(52 \%)$ & $28(56 \%)$ & 0.6892 \\
Primigravida & $24(48 \%$ & $22(44 \%)$ & \\
Multigravida & \multicolumn{2}{c}{ Table 1. Patient Characteristics } \\
\hline
\end{tabular}

Table 1 shows that both the groups were well matched. No significant difference was observed in mean age, mean gestation, initial SBP (Systolic Blood Pressure) and DBP (Diastolic Blood Pressure) level, booking status and parity at the time of allocation in labetalol and hydralazine group. This mitigate any significant contribution in discrimination.

\begin{tabular}{|cccc|}
\hline Parameter & Labetalol & Hydralazine & p \\
Time(minutes) [Mean \pm SD] Number of doses & $26.04 \pm 11.06$ & $22.20 \pm 12.34$ & 0.1134 \\
1 & $13(26 \%)$ & $17(35 \%)$ & \\
$2-3$ & $25(50 \%)$ & $23(46 \%)$ & 0.71824 \\
4 & $10(20 \%)$ & $7(14 \%)$ & \\
Persistent severe hypertension & $2(4 \%)$ & $3(6 \%)$ & \\
\hline Table 2. Time and Number of Doses Required & \\
to Achieve Target Blood Pressure \\
\hline
\end{tabular}

Table 2 shows that the mean time required to achieve target blood pressure in the labetalol group was 26.04 minutes and in the hydralazine group 2220 minutes. The $p$ value was 0.1134 . Thus the time taken for lowering blood pressure in both the labetalol and hydralazine group were similar and no statistically significant difference was observed. The table shows that majority of the patients required a maximum of 2-3 doses to achieve satisfactory blood pressure control (50\% in labetalol group and $46 \%$ in hydralazine group). $4 \%$ of patients in labetalol group and $6 \%$ in hydralazine group had persistent severe hypertension. However, no statistically significant difference was observed between the two groups.

\begin{tabular}{|cccc|}
\hline Outcome & Labetalol & Hydralazine & p Value \\
Still birth & $1(2 \%)$ & $0(0 \%)$ & 0.3173 \\
Fetal distress & $4(8 \%)$ & $2(4 \%)$ & 0.4142 \\
APGAR<6 & $13(26 \%)$ & $10(20 \%)$ & 0.5316 \\
NICU admission & $20(40 \%)$ & $16(32 \%)$ & 0.5049 \\
\hline \multicolumn{4}{c}{ Table 3. Perinatal Outcome } \\
\hline
\end{tabular}

Table 3 shows that there was 1 stillbirth in labetalol group. No stillbirth was encountered in hydralazine group. foetal distress occurred in 4 cases(8\%) in the labetalol group and 2 cases (4\%) in the hydralazine group. $26 \%$ babies in the labetalol group and $20 \%$ babies in the hydralazine group had APGAR score of less than 6 at birth. $40 \%$ babies in labetalol group and $32 \%$ in hydralazine group required NICU admission. The perinatal outcome in between the two groups were analysed and no statistically significant difference was found.

\begin{tabular}{|cccc|}
\hline Complication & Labetalol & Hydralazine & p Value \\
Headache & $3(6 \%)$ & $13(26 \%)$ & 0.0124 \\
Nausea/vomiting & $2(3 \%)$ & $2(4 \%)$ & 1.0 \\
Dizziness & $3(6 \%)$ & $5(10 \%)$ & 0.4795 \\
\hline \multicolumn{4}{c}{ Table 4. Maternal Complications } \\
\hline
\end{tabular}

Complications observed between labetalol and hydralazine group were similar and no statistical difference was present in terms of nausea/vomiting and dizziness. Dizziness was more common in the hydralazine group $10 \%$ and $6 \%$ in hydralazine and labetalol group respectively). No statistical difference was noted. However, headache was more frequently encountered in patients given hydralazine (26\%) compared to labetalol (6\%) which was statistically significant ( $\mathrm{p}$ value 0.0124 ).

\begin{tabular}{|c|c|c|c|}
\hline $\begin{array}{c}\text { Pulse Rate } \\
\text { (Beats/Minute) }\end{array}$ & $\begin{array}{c}\text { Before } \\
\text { Treatment }\end{array}$ & $\begin{array}{c}\text { After } \\
\text { Treatment }\end{array}$ & p Value \\
\hline Labetalol & $88.28 \pm 4.04$ & $89.24 \pm 3.18$ & 0.1898 \\
\hline Hydralazine & $90.08 \pm 3.77$ & $109.76 \pm 8.26$ & 0.0001 \\
\hline
\end{tabular}

Data is expressed in mean and standard deviation. The table shows that the mean pulse rate before administration of labetalol was $88.28 \pm 4.04$ and after was $89.24 \pm 3.18$. The data was not statistically significant. Whereas, the mean maternal pulse rate before hydralazine therapy was $90.08 \pm 3.77$ and after therapy was $109.76 \pm 8.26$. There was highly significant difference in maternal pulse rate in case of hydralazine therapy whereas no such difference was observed in labetalol group.

\section{DISCUSSION}

The randomized clinical trial conducted by us showed that both hydralazine and labetalol are effective and rapid antihypertensive agents which can be used in severe preeclampsia. Our findings are consistent with studies done beforehand including the Cochrane review, ${ }^{[5]}$ on the efficacy of the drugs in managing hypertensive crisis in pregnancy. In our study the mean age of patients was 24.78 in labetalol group and 24.86 in hydralazine group and there was no significant difference in age distribution which was consistent with the findings in studies conducted by Mabie WC et al,[6] Bhorat IE et al.[7] In study done by Vigil-de-Gracia $P$ et al ${ }^{[8]}$ mean age in labetalol group was 31.3 as compared to 29.9 in hydralazine group. In our study majority of the patients in both arms of the study were primiparas-the finding corroborates with studies done throughout the world by different researchers. The mean gestational age in our study was 36.88 in labetalol group and 37.06 in hydralazine group as majority of the patients presented at 37 weeks or more in both the groups $(64 \%$ and $70 \%$ in labetalol and hydralazine group respectively). The difference was not statistically significant. Similar distribution of mean gestational age was found in the study conducted by Ashe RG et al.[9] 
The average time taken to decrease blood pressure in labetalol group was 26.04 minutes whereas it was 22.20 minutes in hydralazine group. No significant difference was observed in relation to time between the two groups. Our result corroborated with the findings of Nombur LI et al[10] where on an average 40 minutes were required for achieving desired blood pressure control. However, contrary to our finding, the study by Mabie WC et al[6] showed significant intergroup difference in respect to blood pressure control (55.1 minutes in labetalol group and 75.8 minutes in hydralazine group). The number of doses required to achieve target blood pressure were found to be similar in both the groups in our study. This finding was in keeping with previous studies conducted by Vigil de Gracia $\mathrm{P}$ et al, ${ }^{[8]}$ Delgado de Pasquale $S$ et al[11] and Nombur LI et al.[10] In study conducted by Magee LA et al[12] hydralazine was found to be more effective antihypertensive than labetalol.

There was no maternal hypotension in both the groups. There were no significant differences in perinatal outcome in both the groups. In our study, we found $8 \%$ cases of foetal distress in labetalol group and $2 \%$ cases in hydralazine group. The bradycardia observed in hydralazine group occurred after successful lowering of blood pressure and was transient in nature and returned to normal by intravenous fluid infusion, changing mother's posture to left lateral position and moist oxygen inhalation. Whereas, in labetalol group 1 patient showed persistent bradycardia not corrected by intravenous fluid or moist oxygen. Immediate caesarean section was done. However, the other 3 patients demonstrated foetal heart rate deceleration during induction of labour and occurred approximately 4-5 hours after achieving satisfactory blood pressure control. Similar finding was seen in study by Ashe RG et al where $30 \%$ of the cases treated with labetalol showed foetal distress. ${ }^{[9]}$ In study by Garden A et al,[13] all the patients treated with labetalol showed foetal distress, most probably attributed to reduced uteroplacental flow following successful lowering of blood pressure and presence of prematurity and IUGR complicating the pregnancies. There was no stillbirth in hydralazine group. 1 incidence of fresh stillbirth was found in labetalol group. There was placental abruption and huge retroplacental clot was observed following delivery in this case.

We observed significantly higher frequency of headache in patients treated with hydralazine than labetalol $(26 \%$ vs $6 \%$ ). Similar findings were observed in study done by Nombur LI et al.[10] No statistically significant difference in frequency of headache found in study by Vigil-de-Gracia P et el.[8] Similar maternal adverse side effects with either hydralazine or labetalol had been reported by other researchers.[11]

Our study showed a significant increase in mean maternal pulse rate following hydralazine therapy whereas no effect was noted with labetalol. Our finding correlates with studies conducted by Ashe RG et al,[9] Vigil-de-Gracia $\mathrm{P}$ et al[8] and Harper A et al.[14] In our study 84\% patients in hydralazine group presented with tachycardia. However, our finding of maternal pulse rate in labetalol group was in stark contrast with study by Bhorat IE et al[7] where a significant fall in maternal pulse rate occurred following labetalol administration.

\section{CONCLUSIONS}

Both hydralazine and labetalol appear to be equally effective in achieving desired level of blood pressure. The time taken and number of doses required to lower blood pressure to target level were almost similar in both the groups. Hydralazine was associated with significantly higher maternal side effects like tachycardia, palpitations and headache. The foetal complications were similar in each group. Patients tolerated the maximum doses of hydralazine and labetalol well. No drug discontinuation was required due to major side effects. Availability of parenteral hydralazine serves as an alternative management in acute hypertension in pregnancy, besides labetalol.

\section{ACKNOWLEDGEMENT}

We would like to thank Prof. Dr. Shyamapada Pati for his affectionate guidance, help and assistance, Dr. Md. Aftabuddin Mondal for his continuous inspiration, Mr. Asif Ahmed Siddik for his technical help, friendly advice and sheer efficiency in helping us to get this work done.

\section{REFERENCES}

[1] Cunningham FG, Leveno KJ, Bloom SL, et al. William's Obstetrics. Chap - 40. Hypertensive disorders. 24th edn. New York: McGraw-Hill Medical Publishing Division 2014: p. 728.

[2] Clark SL, Hankins GDV. Preventing maternal deaths: 10 clinical diamonds. Obstet Gynecol 2012;119(2 Pt 1):3604.

[3] NHBPEP Working Group on High Blood Pressure. Report of NHBPEP Working Group in Hyperte Royal College of Obstetrics and Gynecology. The management of severe preeclampsia/eclampsia. Evidence bases clinical (Guideline No 10(A)).

[4] ACOG Committee on Obstetric Practice. ACOG Practice Bulletin. Diagnosis and management of preeclampsia and eclampsia. No. 33, January 2002. Reaffirmed 2012b. American College of Obstetricians and Gynecologists. Int J Gynaecil \& Obstet 2002;77(1):67-75.

[5] Duley L, Henderson-Smart DJ, Meher S. Drugs for rapid treatment of very high blood pressure during pregnancy. Cochrane Database Syst Rev 2007;4:1-82.

[6] Mabie WC, Gonzalez AR, Sibai BM, et al. A comparative trial of labetalol and hydralazine in the acute management of severe hypertension complicating pregnancy. Obstet Gynecol 1987;70(3 Pt 1):328-33.

[7] Bhorat IE, Naidoo DP, Rout CC, et al. Malignant ventricular arrhythmias in eclampsia: a comparison of labetalol with dihydralazine. Am J Obstet Gynecol 1993;168(4):1292-6.

[8] Vigil-De Gracia P, Lasso M, Ruiz E, et al. Severe hypertension in pregnancy: hydralazine or labetalol. A randomized clinical trial. Eur J Obstet Gynecol Reprod Biol 2006;128(1-2):157-62. 
[9] Ashe RJ, Moodley J, Richards AM, et al. Comparison of labetalol and hydralazine in hypertensive emergencies of pregnancy. S Afr Med J 1987;71(6):354-6.

[10] Nombur LI, Agida ET, Isah AY, et al. A comparison of hydralazine and labetalol in the management of severe preeclampsia. J Women's Health Care 2014;3(6):200.

[11] De Pasquale SD, Velarde R, Reyes O, Hydralazine vs labetalol for treatment of severe hypertensive disorders of pregnancy. A randomized controlled trial. Preg Hyper: An Int J Women's Card Health 2014;4(1):19-22.
[12] Magee LA, Cham C, Waterman EJ, et al. Hydralazine for treatment of severe hypertension in pregnancy: metaanalysis. BMJ 2003;327(7421):955-60.

[13] Garden A, Davey DA, Dommisse J. Intravenous labetalol and intravenous dihydralazine in severe hypertension in pregnancy. Clin Exp Hypertens B 1982;1(2-3):371-83.

[14] Harper A, Murnaghan GA. Maternal and foetal hemodynamics in hypertensive pregnancies during maternal treatment with intravenous hydralazine or labetalol. $\mathrm{Br}$ J Obstet Gynecol 1991;98(5):453-9. 